\title{
Self-Heating and Reliability-Aware "Intrinsic" Safe Operating Area of Wide Bandgap Semiconductors - An Analytical Approach
}

\author{
Bikram Kishore Mahajan, Yen-Pu Chen, Nicolò Zagni and Muhammad Ashraful Alam*
}

\begin{abstract}
The emergence of several technology options and the ever-broadening range of applications (e.g., automotive, smart grids, solar/wind farms) for power electronic devices suggest both a need and an opportunity to develop unifying principles to guide the development of wide bandgap (WBG) semiconductors. Unfortunately, power electronic devices are typically evaluated with a variety of elementary figure of merits (FOMs), which offer inconsistent/contradictory projections regarding the relative merits of emerging technologies. Indeed, one relies on the empirical (extrinsic) safe-operating area (SOA) of a packaged device to ultimately assess the performance potential of a technology option. Unfortunately, extrinsic SOA can only be calculated a posteriori, i.e., after precise measurement of the fabricated device parameters, making it suitable only for relatively mature technologies. Based on the insights of material-devicecircuit-system performance analysis of a variety of idealized WBG power electronic devices (e.g., GaN HEMT, $\beta-\mathrm{Ga}_{2} \mathrm{O}_{3}$ MOSFET), in this paper, we analytically derive a comprehensive, substrate-, self-heating-, and reliability-aware "intrinsic/limiting" safe operating area (SOA) that establishes $a$ priori, i.e., before device fabrication, the optimum and self-consistent trade-off among breakdown voltage, power consumption, operating frequency, heat dissipation, and reliability. We establish the relevance of the intrinsic-SOA by comparing its prediction with a broad range of experimental data available in the literature. In between the traditional FOMs and extrinsic SOA, the intrinsic SOA allows fundamental/intuitive re-evaluation of intrinsic technology potential for power electronic devices and identifies specific performance bottlenecks and how to circumvent them.
\end{abstract}

Index Terms - Power Electronics, Safe Operating Area, Selfheating, Reliability, Thermal Resistance, Figure of Merit.

\section{INTRODUCTION}

A $\mathrm{n}$ experimentally measured safe operating area (SOA) diagram of a packaged and fully processed device ultimately defines the potential and guides the development of power electronics devices [1]. The SOA of a MOSFET refers to voltage and current limits within which the device can be safely operated. Fig. 1 shows a typical SOA defined by the following limits: on-resistance $\left(R_{o n}\right)$, breakdown voltage $\left(V_{b d}\right)$, maximum current $\left(I_{\max }\right)$, and maximum power $\left(P_{\max }\right)$. The SOA deviates from its ideal form due to its thermal and reliability constraints and depends on serval device parameters such as ambient temperature $\left(T_{a m b}\right)$, switching frequency $(f)$,

B. K. Mahajan, Y.-P. Chen, and M. A. Alam are with Department of Electrical and Computer Engineering, Purdue University, West Lafayette, IN 47907 USA. (e-mail: alam@purdue.edu). B. K. Mahajan and Y.-P. Chen contributed equally to this study.

N. Zagni, is with DIEF Department, University of Modena and Reggio Emilia, 41125, Modena, Italy. duty cycle $(D)$, thermal resistance $\left(\theta_{t h}\right)$, etc., which must be considered in order to construct an accurate device SOA. For example, operating at a higher ambient temperature decreases $I_{\text {max }}, P_{\text {max }}$, and $R_{\text {on }}$ limits, thus decreasing the SOA (Fig. 1).

Despite its importance, it is difficult to reliably calculate the extrinsic SOA of emerging WBG semiconductors because of: 1) immature process technology, 2) unknown material parameters, and 3) lack of fully packaged devices. Therefore, in practice, several simple, easy-to-calculate, material-specific FOMs are used to define and compare the device potential of emerging technologies. For example, Baliga FOM (BFOM) [2] establishes a trade-off between $V_{b d}$ and specific on resistance $\left(R_{o n, s p}\right)$, provided that self-heating $(\mathrm{SH})$ is non-existent. Other popular FOMs defined by Keyes [3], Johnson [4], Huang [5], Shenai [6], etc., variously emphasize self-heating, chip-area, operating frequency, charging time, etc., all in an effort to estimate the quantities of interest.

Unfortunately, it is well known that these traditional FOMs often lead to confusing and inconsistent results regarding the performance potential of various emerging technologies [6]. For example, BFOM suggests $\beta-\mathrm{Ga}_{2} \mathrm{O}_{3}$ to be a promising material for power electronics with excellent $V_{b d}$-to- $R_{o n, s p}$ ratio [7], [8]. Yet, self-consistent simulations find the potential of $\beta-\mathrm{Ga}_{2} \mathrm{O}_{3}$ to be compromised by $\mathrm{SH}$ associated with its poor thermal conductivity $(\lambda)$ [9]-[11]. Moreover, different material systems (e.g., $\mathrm{Si}, \mathrm{GaN}, \mathrm{SiC}$ ) and device configurations (e.g. VDMOS, LDMOS, HEMT) degrade differently as a function of voltage and temperature. Therefore, in practice, technology development generally reverts to detailed and time-consuming material-device-circuit-system simulations or empirically measured parameters based on early-stage products to assess performance [10], [13]-[15]. While resorting to this kind of

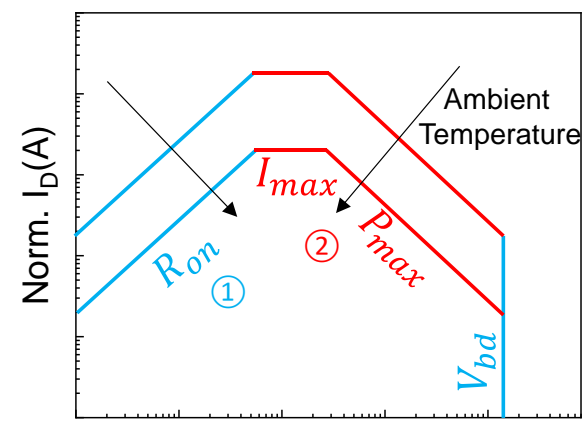

Norm. $\mathrm{V}_{\mathrm{D}}(\mathrm{V})$

Fig. 1. A typical device SOA of a power transistor. It is limited by four quantities: maximum current, maximum power, on-resistance, and breakdown voltage. SOA decreases with increase in the ambient temperature.

Copyright (C) 2021 IEEE. Personal use of this material is permitted. However, permission to use this material for any other purposes must be obtained by sending a request to pubs-permissions@iee.org 
end-to-end analysis is essential for device optimization, the approach might limit the ability to make the right decisions at an early stage of technology development, especially when the transport and thermal models of newly emerging WBG semiconductors, available in TCAD tools, are yet to be vetted against high temperature and high voltage data.

In this paper, we show that recent progress in analytical modeling of the electro-thermal transient response of transistors with multi-layer substrates [16]-[18] as well as the development of generalized multi-hotspot reliability theories of power transistors [19]-[22] allows us to construct an intrinsic SOA ( $i$-SOA) that balances the multi-dimensional trade-off among $V_{b d}, R_{o n, s p}, f$, substrate-aware $\mathrm{SH}$, and reliability. For calculation of $i$-SOA, it is assumed that the material is perfect(i.e., no defects like threading dislocation are present) and that packaging thermal resistance is negligible. Although not a substitute for industry-relevant SOA based on end-to-end modeling and careful qualification of packaged devices, the $i$ SOA obtained here can be used to guard-band vast amount of experimental data reported in the recent literature and to assess the technology potential of WBG semiconductors at the early stage of development and identify the probable bottlenecks.

The paper is arranged as follows. In Sec. II, we rederive the trade-off between $R_{o n, s p}$ and $V_{b d}$ (Fig. 1, (1)) by including the effect of SH and explore its implications. In Sec. III, we derive the expressions for $I_{\max }$ and $P_{\max }$ (Fig. 1, (2)) and evaluate the maximum current for WBG semiconductors based on intrinsic material properties. In Sec. IV, we obtain the substrate-aware $i$ SOA for different WBG semiconductors and systematically compare them with corresponding experimental data. Finally, Sec. V highlights the importance of self-consistent inclusion of transistor degradation. We conclude the paper by discussing the implications of the reliability-aware $i$-SOA for different WBG materials.

\section{Generalized $R_{\mathrm{ON}}$ AND $V_{\mathrm{BD}}$ LIMITS}

\section{A. Analytical Model for SH-dependent $R_{\text {on }}$ and $V_{b d}$}

Initially proposed by Adler and Westbrook [23], the power electronics FOMs have traditionally focused on reducing $R_{o n, s p}$, for a given $V_{b d}$. From BFOM [2], $R_{o n, s p}$ is given by:

$$
B F O M=\frac{V_{b d}^{2}}{R_{o n, s p}}=\varepsilon \mu_{n} E_{C}^{3} / 4 .
$$

where $\varepsilon$ is the permittivity, $\mu_{n}$ is channel mobility and $E_{C}$ is the critical electric field. BFOM for lateral devices can be written alternatively as [24]:

$$
B F O M_{\text {lateral }}=\varepsilon \mu_{n} E_{C}^{3} .
$$

It is to be noted that $\mu_{n}$ is temperature- and dopingdependent. This dependence not only stems from the ambient temperature but also from $\mathrm{SH}$ associated with the operating conditions. The generalized expression is given by [25]:

$$
\mu_{n}\left(T_{o n}\right)=\frac{\mu_{0}}{1+\left(\frac{N_{D} \xi\left(T_{o n}\right)}{N_{\text {ref }}}\right)^{\beta}}\left(\frac{T_{o n}}{300}\right)^{-\gamma} .
$$

where $T_{o n}$ is the temperature at on-state, $N_{D}$ and $N_{r e f}$ are the doping and the reference doping concentration of the material, $\mu_{0}$ is the intrinsic mobility at $\mathrm{T}=300 \mathrm{~K}, \beta, \gamma>0$ are empirical exponents and $\xi\left(T_{o n}\right)$ is the ionization ratio of the dopants, calculated from the occupation probability function as (assuming n-type doping):

$$
\xi\left(T_{o n}\right)=\frac{-1+\sqrt{1+4 g_{D} \frac{N_{D}}{N_{C}} \exp \left(\frac{\Delta E_{D}}{k T_{o n}}\right)}}{2 g_{D} \frac{N_{D}}{N_{C}} \exp \left(\frac{\Delta E_{D}}{k T_{o n}}\right)} .
$$

Here, $\Delta E_{D}$ is doping activation energy, $g_{D}$ is the degeneracy factor, $N_{C}$ is effective density of state in the conduction band. The optimum doping concentration, $N_{D}$ and $V_{b d}$ are determined at off-state, at the temperature $T_{o f f}$, so that,

$$
N_{D} \xi\left(T_{o f f}\right)=\frac{\varepsilon E_{C}^{2}}{2 q V_{b d}} .
$$

where the critical electric field, given by the empirical relation, $E_{C}=E_{C 0}\left(1+\beta^{\prime} T_{o f f}\right)$, increases with the temperature. The coefficient $\beta^{\prime}$ accounts for the decreased impact ionization rate at higher temperatures. Finally, the on-resistance at a temperature $T_{o n}$, is given by:

$$
R_{o n, s p}\left(T_{o n}\right)=\frac{L_{D}}{q \mu_{n}\left(T_{o n}\right) N_{D} \xi\left(T_{o n}\right)} .
$$

where $L_{D}$ is the drift length and $V_{b d}=E_{C} L_{D} / 2$ (assuming nonpunch-through). Putting Eq. (3), (5), (6), we get:

$$
R_{o n, s p}\left(T_{o n}\right)=\frac{4 V_{b d}^{2}}{\varepsilon \mu_{0} E_{C}^{3}}\left[1+\left(\frac{N_{D} \xi\left(T_{o n}\right)}{N_{r e f}}\right)^{\beta}\right]\left(\frac{T_{o n}}{300}\right)^{\gamma} \frac{\xi\left(T_{o f f}\right)}{\xi\left(T_{o n}\right)}
$$

We will see below that Eq. (7) establishes a novel and essential temperature-dependent generalization of $R_{o n, s p}$, and thus Eq. (1). Unlike the traditional FOMs, $R_{o n, s p}\left(T_{o n}\right)$ in Eq. (7) analytically accounts for excess SH through its impact on mobility, dopant ionization ratio, and impact ionization rate. Previously, one needed to solve multiple equations numerically to include these effects [26]. The idealized bulk parameters in Eq. (7) are easily obtained from online databases (e.g., Ioffe Institute's semiconductor repository [27], first-principle calculation [28]-[30], bulk-measurements [9], [31], [32], and/or TCAD simulations [10], [33]-[35]).

\section{B. Counterbalancing Roles of Self-Heating in $R_{o n, s p}$ and $V_{b d}$}

For typical switching speeds ( $f>1 \mathrm{kHz}), T_{o n} \sim T_{\text {off }}$ because the thermal time constant of the whole system is large enough to avoid significant cooling during the on-to-off transition. For fully ionized materials with $\Delta E_{D} \sim k T_{A}$, Eq. (7) predicts $\xi\left(T_{o f f}\right) / \xi\left(T_{o n}\right) \sim 1$, so that SH increases $R_{o n, s p}\left(T_{o n}\right)$ through mobility degradation. However, for materials (e.g. Diamond) with deep dopants (i.e., $\Delta E_{D} \gg k T_{A}$ ), the $\mathrm{SH}$ ionizes more dopants, so that $\xi\left(T_{o n}\right)>\xi\left(T_{o f f}\right)$. By Eq. (7), $R_{\text {on }, s p}\left(T_{o n}\right)$ is determined by the balance between reduced mobility and increased doping. Similarly, the SH-supported 
(a)

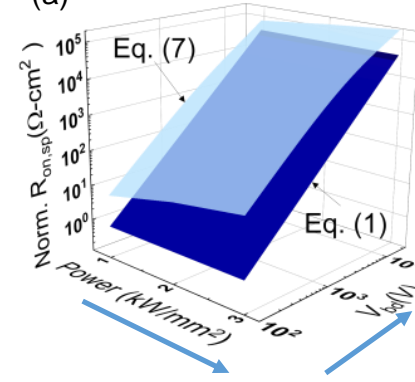

(b)

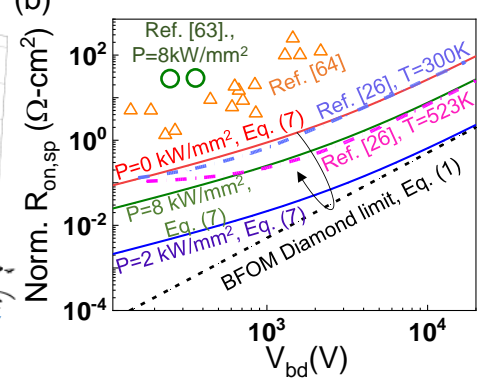

Fig. 2. (a) While Eq. (1) is constant w.r.t. power, $R_{o n, s p}\left(T_{o n}\right)$ (for different power, P) varies depending on the degree of self-heating. As input power increases in a Si device, SH increases as reflected in the curved contour in $R_{o n, s p}\left(T_{o n}\right)$. The blue arrows indicate the direction of increasing values (b) $R_{o n, s p}\left(T_{o n}\right)$ vs. $V_{b d}$ for Diamond. The solid lines show $R_{o n, s p}\left(T_{o n}\right)$ [Eq. (7)] for different applied power. BFOM for Diamond is given as the grey dotted line. At T=300K and 523K, Eq. (7) matches the numerical results from [26], shown as dashed-dotted lines. The black arrow shows the turn-around for Diamond. Data from [63], [64] are plotted as well for reference.

reduction of impact ionization rate increases the critical field and $V_{b d}$.

Fig. 2(a) shows that SH reduces the performance potential of Si because of mobility degradation. Remarkably, this is not the case for diamond, as shown in Fig. 2(b). When the power dissipation $\left(P_{D}\right)$ increases from $0 \mathrm{~kW} / \mathrm{mm}^{2}(\mathrm{~T}=300 \mathrm{~K})$ to 8 $\mathrm{kW} / \mathrm{mm}^{2}(\mathrm{~T} \sim 525 \mathrm{~K})$, the increased ionization overcomes mobility degradation, leading to improved $R_{o n, s p}$ [26]. Diamond benefits from SH because of its higher ionization energy which is otherwise determined by its larger bandgap and its lower dielectric constant (e.g., Boron is an acceptor level with $E_{A}=0.37 \mathrm{eV}$ [36]). The increasing conductivity with $P_{D}$ reduces $R_{o n, s p}\left(T_{o n}\right)$. Note that Ref. [26] reaches similar conclusions through numerical simulations at increased ambient temperature, however, our work emphasizes the role of self-heating in achieving similar performance gain even at typical ambient temperature. However, increasing $P_{D}$ beyond a critical value may lead to a reduction in $R_{o n, s p}\left(T_{o n}\right)$ because of increased mobility degradation that eventually counterbalances the beneficial effects of SH (and reduces the gap between the experimental data shown by symbols and the corresponding performance limit). In short, Fig. 2 demonstrates the counterintuitive role of operating conditions in shaping the ultimate performance of power devices and the ability of $R_{o n, s p}\left(T_{o n}\right)$ to capture the trend.

\section{Substrate-Aware $R_{o n, s p}$}

According to Eq. (7), the performance of materials such as $\beta-\mathrm{Ga}_{2} \mathrm{O}_{3}$, with high $E_{C}$, but with low thermal conductivity, may suffer due to SH. Therefore, an ultra-thin layer of $\beta-\mathrm{Ga}_{2} \mathrm{O}_{3}$ must be placed on top of a high thermal conductivity substrate (e.g., sapphire [11], diamond [37], AlN [38]) for efficient heat spreading. While a lot of improvements are required in the fabrication techniques of $\beta-\mathrm{Ga}_{2} \mathrm{O}_{3}$ FETs to emerge as the high performing power electronics substrate as originally promised, bilayer $\beta-\mathrm{Ga}_{2} \mathrm{O}_{3}$ devices should be compared against $R_{\text {on,sp }}\left(T_{\text {on }}\right)$ expression (Eq. (7)) to achieve realistic performance prediction of the technology. $\theta_{t h}$ of the bilayer structures can be estimated using the simple formula provided

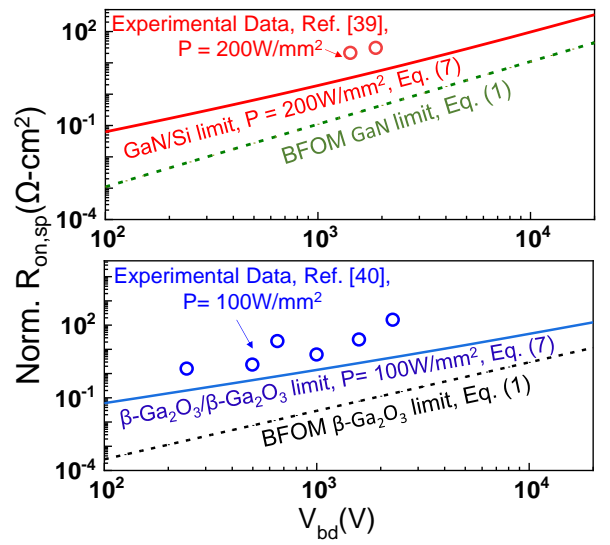

Fig. 3. $R_{o n, s p}\left(T_{o n}\right)$ [Eq. (7)] predicted for bilayer $\mathrm{GaN}$ and $\beta-\mathrm{Ga}_{2} \mathrm{O}_{3}$ structures at $P=1 \mathrm{~kW} / \mathrm{mm}^{2}$ in comparison to the experimental data on bilayer structures. As an example, GaN-on-Silicon (top) and $\beta-\mathrm{Ga}_{2} \mathrm{O}_{3}$-on- $\beta-\mathrm{Ga}_{2} \mathrm{O}_{3}$ device (bottom) have been shown here. Data taken from [39], [40].

in Appendix A. Fig. 3 shows the comparison between the experimental data of a GaN-on-Si device [39] and a $\beta-\mathrm{Ga}_{2} \mathrm{O}_{3}$ on- $\beta-\mathrm{Ga}_{2} \mathrm{O}_{3}$ device [40]. In both cases, the generalized $R_{\text {on,sp }}$ (Eq. (7)) anticipates the experimental trends better than BFOM and suggests that the devices may already be performing close to their intrinsic limits, further indicating that BFOM represents an overly optimistic metric for nontraditional WBG materials.

In practice, the rise of the junction temperature is limited by packaging and other considerations, suggesting an upper limit of power $\left(P_{\max }\right)$ and current $\left(I_{\max }\right)$ that can be sustained by a technology. We will discuss this limiting current performance in the next section.

\section{GENERALIZED $I_{\text {max }}$ AND $P_{\max }$ LIMITS}

\section{A. Analytical Derivations}

Since the device mobility degrades with increasing temperature, the device junction temperature is required to be well below the maximum junction temperature $\left(T_{j, \max }\right)$ to maintain proper functionality. In other words, for a given material, geometry, structure, and package (i.e., given $\theta_{t h}$ ), the maximum allowable power dissipation $\left(P_{\max }\right)$ can be represented as:

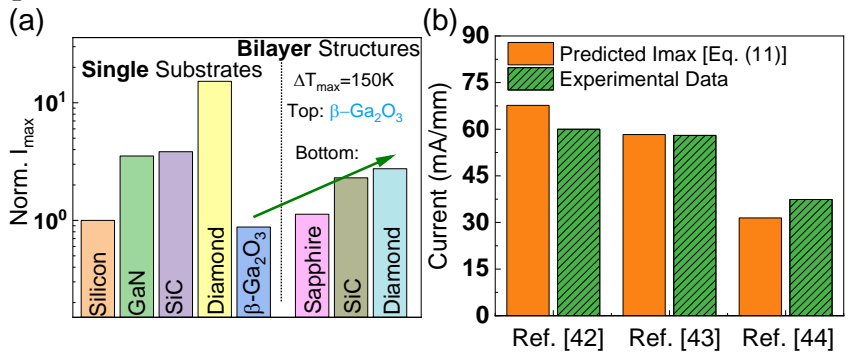

Fig. 4. (a) (Left)Normalized $I_{\max }$ metric for WBG materials at $\Delta T_{\max }=150$ K. (Right) $\beta-\mathrm{Ga}_{2} \mathrm{O}_{3}$ channel layer on various substrates (Sapphire, SiC, Diamond). The performance (i.e., $I_{\max }$ ) can be improved by depositing $\beta$ $\mathrm{Ga}_{2} \mathrm{O}_{3}$ on higher thermal conductivity substrates. The arrow indicates increasing performance of $\beta-\mathrm{Ga}_{2} \mathrm{O}_{3}$ FETs. (b) Comparison of on-current predicted by Eq. (11) with that of experimental data.

$$
P_{\text {max }}=\frac{\Delta T_{\max }}{\theta_{t h, j-c}+\theta_{t h, c-a}}
$$


where $\Delta T_{\max }$ is the maximum temperature rise, $\left(\Delta T_{\max }=\right.$ $\left.T_{j, \max }-T_{a m b}\right), T_{a m b}$ is the ambient temperature, $\theta_{t h, j-c}$ and $\theta_{t h, c-a}$ are junction-to-case and case-to-ambient thermal resistances. $P_{\max }$ can be directly linked to the maximum current rating $\left(I_{\max }\right)$ if only the conduction loss is considered. To determine $I_{\text {max }}$ for a device, $T_{j, \max }$ is fixed, in contrast to the procedure for $R_{o n, s p}$ derivation in Sec. II, where $\Delta T$ was determined by $P$. To determine the intrinsic limiting performance, we neglect $\theta_{t h, c-a}$ (can vary with different package configurations) and switching loss (may vary with different gate drive circuits and switching conditions [5]). Therefore, the maximum allowable current density can be written as:

$$
I_{\text {max }}=\sqrt{\frac{\Delta T_{\max }}{\theta_{t h, j-c} R_{o n}}} .
$$

where $I_{\max }$ is the maximum allowable current in a device at $\Delta T_{\text {max }}$. Let us assume $\theta_{t h, j-c}=\left(W \lambda_{s u b} S\right)^{-1}$, where $W$ is the device width, $\lambda_{\text {sub }}$ is the substrate thermal conductivity, and $S$ is the shape factor [41]. In practice, one can easily obtain the shape factor for a technology from thermal modeling and/or experimental measurements [16]-[17]. Note that effective $\theta_{t h, j-c}$ (for the packaged chip) can change with duty cycle and frequency, as discussed in Section IV. For a lateral device, the normalized $I_{\max }$ can be written as:

$$
\frac{I_{m a x}}{W}=\sqrt{D_{d r}} \sqrt{\frac{\lambda_{s u b} S \Delta T_{m a x}}{R_{o n, s p}\left(T_{o n}\right)}} .
$$

where $D_{d r}$ is the drift region thickness. It can be easily adapted for vertical devices by interchanging $D_{d r}$ with the gate length. For $I_{\max }$ calculations, $T_{o n} \equiv T_{j, \max }$. Substituting the $R_{o n, s p}\left(T_{o n}\right)$ as in Eq. (7), we obtain a metric to compare the maximum current for various WBG semiconductors, as:

$$
\frac{I_{\max }}{W} \propto \sqrt{\frac{\varepsilon \mu_{n} E_{C} \lambda_{\text {sub }} S \Delta T_{\max }}{\frac{\xi\left(T_{o f f}\right)}{\xi\left(T_{o n}\right)}\left(\frac{T_{o n}}{300}\right)^{\gamma}} .}
$$

The corresponding generalization of $I_{\max }$ (Eq. (11)) provides a new limit that explicitly accounts for the first time, the effect of temperature-dependent mobility, shape factor, and incomplete ionization.

\section{B. Substrate-Aware $I_{\text {max }}$ in lateral devices}

The effect of SH is more severe in lateral devices compared to vertical devices, and hence lateral devices are compared for demonstration in various WBG materials. The device dimensions and basic parameters used for calculations are tabulated in Appendix D. $\theta_{t h}$ is calculated considering 3D thermal spreading (formula provided in Appendix B). By alternating the corresponding $\lambda$ for channel and substrate material, the $I_{\max } \mathrm{FOM}$ at fixed $\Delta T_{\max }(150 \mathrm{~K}$ here $)$ for (a)
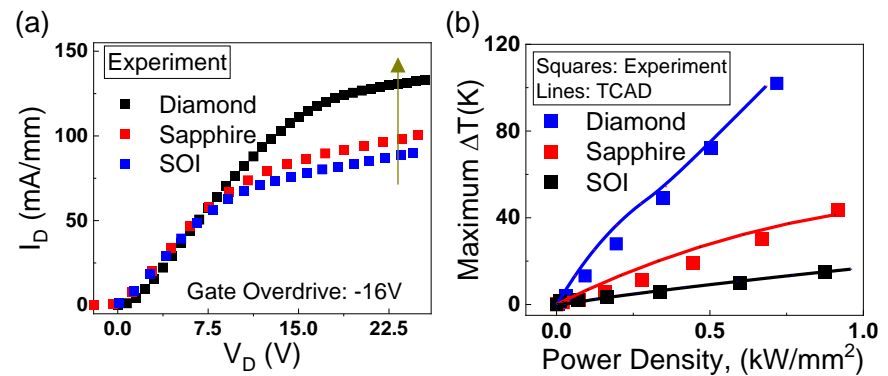

Fig. 5. $\beta-\mathrm{Ga}_{2} \mathrm{O}_{3}$ FETs on various substrates. (a) Increased current in $\beta-\mathrm{Ga}_{2} \mathrm{O}_{3}$ FETs as predicted by $I_{\max }$ equation (eq. 11), [10]. (b) Decreased junction temperature, resulting from the use of higher thermal conductivity substrates.

various WBG materials is easily calculated. Fig. 4 (a) shows the calculated values normalized to the value of $\mathrm{Si}$, including the bulk material (left half). For bulk materials, at the same $\Delta T_{\max }$ $\mathrm{GaN}$ and $\mathrm{SiC}$ have four times larger $I_{\max }$ than Si. Bulk $\beta-\mathrm{Ga}_{2} \mathrm{O}_{3}$, however, performs worse than $\mathrm{GaN}$ and $\mathrm{SiC}$. As shown in Fig. 4 (b), the $I_{\max }$ values predicted by Eq. (11) for $\beta-\mathrm{Ga}_{2} \mathrm{O}_{3}$, reproduces the experimentally obtained current for $\beta-\mathrm{Ga}_{2} \mathrm{O}_{3}$ in Ref. [42], Ref. [43], and Ref. [44] for different geometric configurations. The prediction, by Eq. (11) for Ref. [44] was slightly lower compared to the experimental data, which could be due to the uncertainty $(10-20 \%$ [45]) in the Raman thermography technique used to obtain the peak channel temperature.

To improve $I_{\max }$ (right half, Fig. 4(a)), we consider $\beta-\mathrm{Ga}_{2} \mathrm{O}_{3}$ on various substrates with higher thermal conductivity. The device geometry of the bilayer structure $\left(\beta-\mathrm{Ga}_{2} \mathrm{O}_{3}\right.$ on various substrates) has been tabulated in Appendix D. The $I_{\max }$ metric assigns $\beta-\mathrm{Ga}_{2} \mathrm{O}_{3}$ a lower rank than other WBG materials due to its low thermal conductivity. The bottleneck of the low thermal conductivity can be alleviated by stacking $\beta-\mathrm{Ga}_{2} \mathrm{O}_{3}$ on high thermal conductivity substrates. This is achieved by fabricating $\beta-\mathrm{Ga}_{2} \mathrm{O}_{3}$ FETs on Sapphire and Diamond as shown in Fig. 5(a). As predicted by $I_{\max }$, high current is observed at the same gate overdrive for $\beta-G a_{2} \mathrm{O}_{3}$ FETs on Sapphire and Diamond. This is because of the reduction in junction temperature, as revealed by thermo-reflectance measurements and TCAD simulations (Fig. 5(b)) [10]. Therefore, qualitatively $\beta-\mathrm{Ga}_{2} \mathrm{O}_{3}$ can be a material of choice for power electronics, only if appropriate thermal shunts or other cooling systems are employed.

\section{SElF-HEATING AWARE INTRINSIC SOA FOR WBG SEMICONDUCTORS}

The substrate- and SH-aware expressions obtained in the previous sections can now be used for obtaining the $i$-SOA of a power device. Since frequency dependence is an important consideration for power transistors, the effect of transient thermal resistance $(Z)$ needs to be considered. At $T_{a m b}=300$ $\mathrm{K}$, by setting $T_{j, \max }=450 \mathrm{~K}, P_{\max }$ can be determined using the following relation:

$$
P_{\text {max }}=\frac{T_{j, \max }-T_{a m b}}{\theta_{t h} \times Z_{m}\left(t_{o n}\right)}=I_{D} \times V_{D}
$$




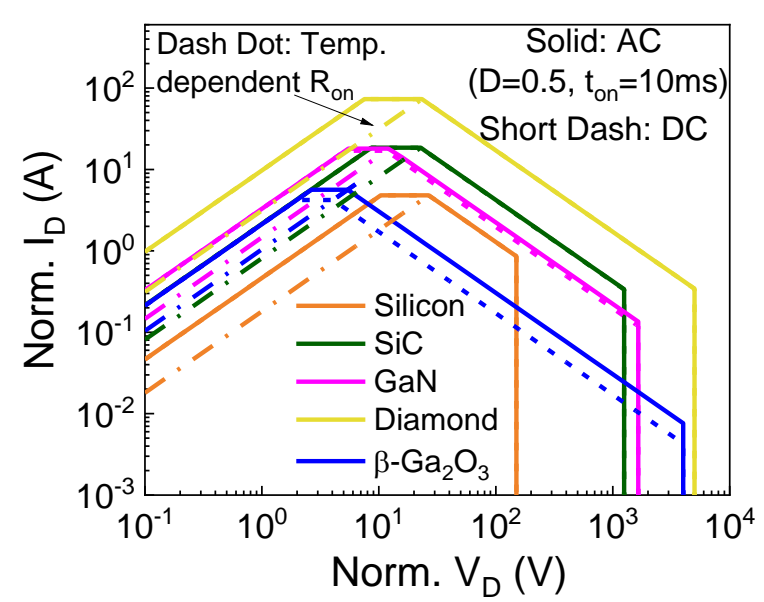

Fig. 6. $i$-SOA predicted for various bulk WBG materials based on Eqs. 10-13.

$\theta_{t h}$ is the DC thermal resistance, $Z_{m}\left(t_{o n}\right)<1$, is the normalized transient thermal impedance, $t_{o n}$ is the pulse duration ( $t_{o n}=D \zeta, \zeta$ is the period, $D$ is the duty cycle). Considering multiple stages of equivalent RC-time constants due to package, submounts, heat sink, PCB, etc., one can write [17], [18]:

$$
\begin{gathered}
Z_{m}\left(t_{o n}\right)=D+(1-D) \sum_{i} \frac{\theta_{t h, i}}{\theta_{t h, t o t}} C_{i}(D, \zeta) Z_{0, i}\left(t_{o n}\right) \\
\text { with } C_{i}(D, \zeta)=\frac{1-\exp \left(-\frac{(1-D) \zeta}{\tau_{t h i}}\right)}{1-\exp \left(-\frac{\zeta}{\tau_{\text {thi }}}\right)} .
\end{gathered}
$$

where index $i$ stands for a certain stage of RC circuit $(i=1$ is the chip itself), $C_{i}(D, \zeta)$ is a correction function that depends on $\tau_{t h}$ of each stage. $Z_{0}$ is the transient thermal impedance for a single pulse expressed as $\left[1-\exp \left(t_{o n} / \tau_{t h}\right)\right]$, where $\tau_{t h}\left(\tau_{t h}=\theta_{t h} C_{t h}\right)$ can be calculated by the parameters given in Appendix D. The derivation of Eq. (13) is provided in Appendix C. This is the first closed-form analytical expression for arbitrary complex package structure subjected to pulses of arbitrary duty cycle that considerably simplifies the calculation of $i$-SOA. Knowing $Z_{m}\left(t_{o n}\right)$, the current limit can be found from the following expression:

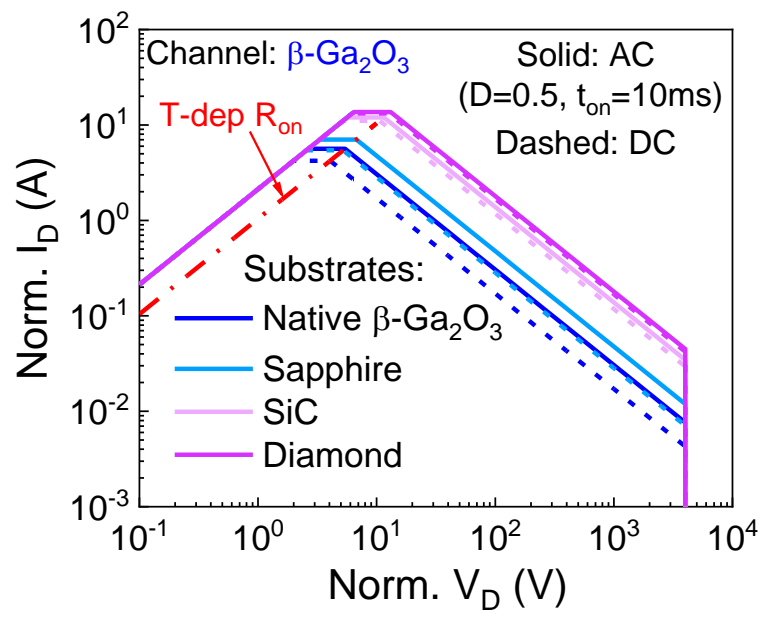

Fig. 7. $i$-SOA predicted for bilayer substrates. $\beta-\mathrm{Ga}_{2} \mathrm{O}_{3}$ on various substrates (native $\beta-\mathrm{Ga}_{2} \mathrm{O}_{3}$, Sapphire, $\mathrm{SiC}$, Diamond) are shown as an example. Temperature-dependent $R_{\text {on }}$ has been shown in red dashed lines.

$$
I_{\text {max }}=\sqrt{\frac{T_{j, \max }-T_{a m b}}{\theta_{t h} Z_{m}\left(t_{o n}\right) \times R_{o n}}} .
$$

Using Eqs. 7, 11-14, the $i$-SOA for different bulk WBG materials (Fig. 6) and bilayer structure (Fig. 7, $\beta-\mathrm{Ga}_{2} \mathrm{O}_{3}$ as an example) can be calculated analytically by assuming $t_{\text {on }}=10$ $\mathrm{ms}$ and $D=0.5$ for a standalone device $(i=1)$. Using the same procedure, the performance and $i$-SOA of other device systems (e.g. GaN-on-Si, $\mathrm{GaN}-$ on-SiC, etc.) can be easily evaluated. In Fig. 6, some of the materials (except $\mathrm{GaN}$ and $\beta-\mathrm{Ga}_{2} \mathrm{O}_{3}$ ) show a similar DC and $\mathrm{AC}$ response because of a low time constant. It is evident that device SH plays an important role in determining performance. Because of larger $\theta_{t h}, \beta-\mathrm{Ga}_{2} \mathrm{O}_{3}$ gains more $i$-SOA in AC operations, compared to DC condition. We reach similar conclusions for other WBG materials as well. In Fig. 6, materials with larger $\lambda_{\text {sub }}$ have larger $i$-SOA, as expected. Thus, the $i$-SOA obtained here gives a holistic devicecircuit-system perspective of WBG semiconductor performance.

\section{RELIABILITY-AwARE SOA}

\section{A. Reliability-Aware $R_{o n, s p}$}

Several previous works emphasize the importance of defining a reliability-aware generalization of the SOA [46], [47]. The reliability-aware SOA is defined in various ways viz., voltage-dependent HCD stress SOA [48], thermal SOA [49], catastrophic failure SOA [50], etc. Although achievable for extensively studied devices (such as Si LDMOS), these SOAs are difficult to be determined for emerging WBG materials. Therefore, we propose a reliability-aware $i$-SOA that can be analytically obtained for a very well-researched device like $\mathrm{Si}$ LDMOS, as well for state-of-the-art emerging WBG materialbased devices like Diamond and $\beta-\mathrm{Ga}_{2} \mathrm{O}_{3}$.

We note that $R_{o n, s p}$ degrades over stress time (t) as bulk and interface traps are generated during device operation. These traps scatter electrons and reduce mobility. The exact form of the mobility degradation depends on specific material; however, it can be simply expressed in the phenomenological Sun-Plummer form [51]:

$$
\mu_{n}\left(T_{o n}, t\right)=\frac{\mu_{n}\left(T_{o n}, t=0\right)}{\left(1+\alpha \Delta N_{I T}(t)\right)}
$$

and time evolution of $\Delta N_{I T}(t) \sim f(t / \tau)$ depends on the voltage- and temperature-dependent scaling factor, $\tau$ [52], [53]. The precise functional form (e.g., power-law, exponential, sigmoid, etc.) of $N_{I T}$ generation depends on the material of choice. The reliability factor appears as a multiplicative derating factor [54]-[56] in Eq. (7) for $R_{\text {on,sp }}$, namely:

$$
R_{o n, s p}^{*}\left(T_{o n}, t\right)=R_{o n, s p} \times T_{f} \times R_{f}(t)
$$

where $T_{f}$ is the temperature correction factor, and $R_{f}$ is the reliability correction factor, given as:

$$
T_{f}=\left[1+\left(\frac{N_{D} \xi\left(T_{\text {on }}\right)}{N_{\text {ref }}}\right)^{\beta}\right]\left(\frac{T_{\text {on }}}{300}\right)^{\gamma} \frac{\xi\left(T_{\text {off }}\right)}{\xi\left(T_{\text {on }}\right)}
$$


(a)

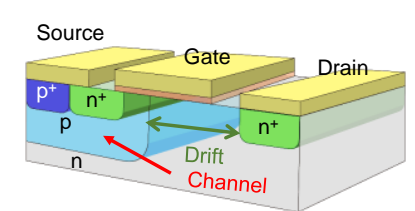

(c)

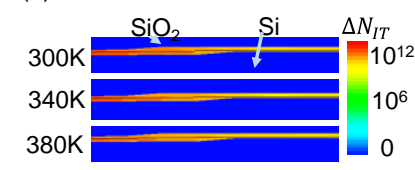

(e)

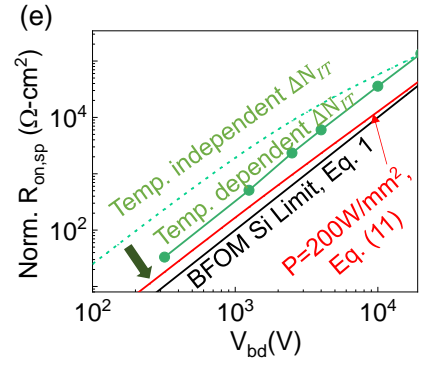

(b)

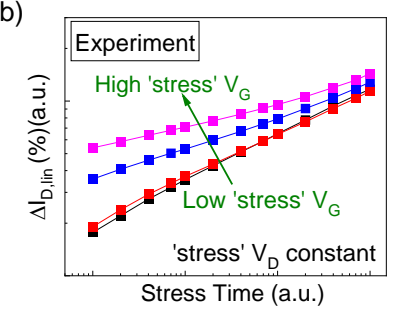

(d) Squares: Drift

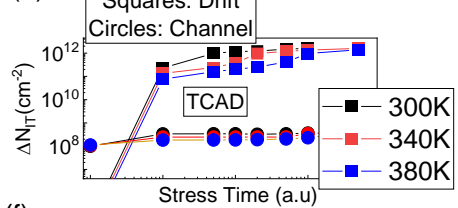

(f)

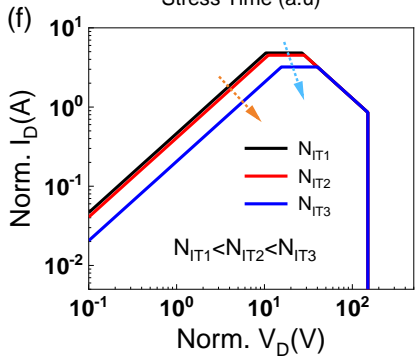

Fig. 8. (a) Schematic of the Si LDMOS used in the study. (b) TCAD calibration of the temperature dependent HCD characteristics of the LDMOS. (c) The $2 \mathrm{D}$ plots showing the $\mathrm{Si} / \mathrm{SiO}_{2}$ interface and trap generation in the interface, in a Si LDMOS. (d) $\Delta N_{I T}$ with increasing stress time at different temperatures. (e) Reliability-aware $R_{o n, s p}$ or $R_{o n, s p}^{*}$ predicted for Silicon power devices (LDMOS) (f) Reliability-aware $i$-SOA predicted for LDMOS device at different interface trap concentrations.

$$
R_{f}(t)=\left(1+\alpha \Delta N_{I T}(t)\right)
$$

The reliability of power transistors depends on various degradation mechanisms (e.g., HCD [20], BTI [57], the inverse piezoelectric effect [58], etc.) with temperature and voltagedependent degradation rates. The localized generation of $N_{I T}$ in a multi-hotspot power transistor is phenomenologically written as [19], [21]:

$$
\Delta N_{I T}(t)=\frac{A t^{n}}{1+B t^{n}}
$$

where the coefficients $A$ and $B$ may have positive or negative temperature coefficients and power-law or exponential voltage acceleration factors.

\section{B. Reliability-aware intrinsic SOA}

For an illustrative example, the role of trap-induced degradation is analyzed with TCAD simulations on Si LDMOS (Fig. 8 (a)) [59]. The LDMOS has been electrically stressed to analyze the Hot Carrier Degradation (HCD), as shown in Fig 8(b) [19]. In $\mathrm{Si}$ transistors, it is well known that $\Delta N_{I T}(t)$ reduces at higher temperature [56] (Figs. 8(c) and 8(d)) due to reduction of impact ionization rate due to increased phonon scattering. By including reliability aspects connected to $\Delta N_{I T}(t)$ in the $R_{o n, s p}$ expression, one can project device performance over prolonged operation. This is shown in Fig. 8(e), where $R_{o n, s p}^{*}\left(T_{o n}, t\right)$ for $\mathrm{Si}$ LDMOS is calculated with $\Delta N_{I T}(t)$ after certain stress time. As expected, $R_{o n, s p}^{*}\left(T_{o n}, t\right)$ increases due to stress but, at the same time, $\mathrm{SH}$ reduces

$\Delta N_{I T}(t)$ (at a fixed stress time) in this case. This is shown by the dotted (solid) line that represents the impact of $\Delta N_{I T}(t)$ on $R_{\text {on }, s p}\left(T_{\text {on }}\right)$ without (with) thermal effects. The increasing $\mathrm{SH}$ reduces HC-induced $\Delta N_{I T}(t)$, with a small improvement in $R_{o n, s p}^{*}\left(T_{o n}, t\right) . I_{\text {max }}$ accounts for $\Delta N_{I T}(t)$ through $R_{o n, s p}\left(T_{o n}\right)$ and the newly calculated $I_{\max }$ can be used to obtain a Reliability-aware $i$-SOA (Fig. 8(f)). As the stress time increases, $N_{i t}(t)$ increases, thus decreasing the mobility (captured using Eq. (17)), leading to increased $R_{o n, s p}$, decreased $I_{\max }$, and closure of $i$-SOA. Following similar approaches, $i$-SOA can be obtained for other WBG semiconductors as well, provided their stress-dependent $N_{I T}$ is known either by TCAD simulations or experiments.

\section{DISCUSSION AND CONCLUSIONS}

In this paper, we have presented an analytical model (Eqs. 7, 11-14) to calculate the $i$-SOA for modern power electronic devices that accounts for the $\mathrm{SH}$ effect and trap-induced degradation and compared the results with a broad range of experimental data published in the recent literature related to emerging WBG materials, such as $\mathrm{Ga}_{2} \mathrm{O}_{3}$. We have demonstrated that starting with the generalization of BFOM, one can get the device $i$-SOA by carefully considering the $\mathrm{SH}$ effects through substrate-aware $\theta_{t h}$, temperature-dependent mobility, incomplete ionization, and trap generation rate. To account for the substrate-aware $\mathrm{SH}$, accurate modeling of $\theta_{t h}$ is essential, which has been achieved using simple, but robust $\theta_{\text {th }}$ formula (Appendix A and B). These generalizations allow calculating more realistic intrinsic device $i$-SOA, which was previously possible only through end-to-end modeling based on device-specific parameters and/or careful measurement of packaged devices.

1. The temperature- and reliability-aware $i$-SOA reveals that intrinsically diamond is the best possible candidate at high temperature (here $T_{j, \max }=450 \mathrm{~K}$ ) among the $\mathrm{WBG}$ materials surveyed here. The $i$-SOA provides the new insight that Diamond would benefit from $\mathrm{SH}$ and its performance would increase with increasing $P_{D}$, but only until all its dopants are fully ionized, after which the temperature-dependent mobility degradation dictates the performance limit.

2. Similarly, from the perspective of $i$-SOA, $\mathrm{GaN}$ and $\mathrm{SiC}$ have comparable performance, with $V_{b d}$ in the $\mathrm{kV}$ range. In practice, the material quality may further limit the empirical SOA for these materials.

3. Moreover, with proper thermal management (high thermal conductivity substrates, cooling, etc.), $i$-SOA suggests that $\beta-\mathrm{Ga}_{2} \mathrm{O}_{3}$ FETs may be able to deliver performance comparable to $\mathrm{GaN}$ and $\mathrm{SiC}$.

In practice, nonideal effects, such as threading dislocations, interfacial thermal resistance, the choice of substrate, etc. may make the extrinsic SOA somewhat (or substantially) different. Regardless, based on application-specific requirements on 
power dissipation and reliability, the $i$-SOA provides a unifying conceptual framework to assess the early-stage experimental data and to define the accurate merits and fundamental bottlenecks of emerging WBG technologies for specific power electronics applications long before the devices are ready to be packaged and tested.

\section{APPENDIX}

\section{A. Determining Thermal Resistance of a Bilayer Structure}

The self-heating at the device level (i.e., $\Delta T=P_{D} \theta_{t h}$ ) is determined by calculating the $\theta_{t h}$ associated with the structure. While the formula and techniques of determining $\theta_{t h}$ of a bulk substrate is well established, $\theta_{t h}$ of a multi-layered device is more complicated due to the change in the heat spreading angle in different layers.

For a bulk substrate, the simplest approach of calculating $\theta_{t h}$ is to assume that heat spreads at a constant angle, $\omega$ (consider $\omega \sim 45^{\circ}$, for simplicity). For a disk-shaped heat source of radius, $a$, thermal conductivity, $\lambda$, on a substrate of thickness, $\ell$, as shown in Fig. A1(a), $\theta_{t h}$ can be obtained as [60]:

$$
\theta_{\text {th,single layer }}=\frac{1}{\lambda \pi a} \frac{\ell}{(a+\ell \tan \omega)}
$$

Now, let us consider a bilayer substrate with the thickness of the top layer, $\ell_{1}$ and bottom layer, $\ell_{2}$ respectively. The heat spreading angle and the thermal conductivity of the top layer is given as $\omega_{1}$ and $\lambda_{1}$ and correspondingly $\omega_{2}$ and $\lambda_{2}$ for the bottom layer, as shown in Fig. A1(b). Let us also assume that $\lambda_{1}<\lambda_{2}$, because a low thermal conductivity channel is placed on top of a high thermal conductivity substrate for highperformance power electronics devices [11]. To extend Eq. (A1) to a bilayer substrate, we consider a constant spreading angle $\alpha$ for both layers and rewrite Eq. (A1) as:

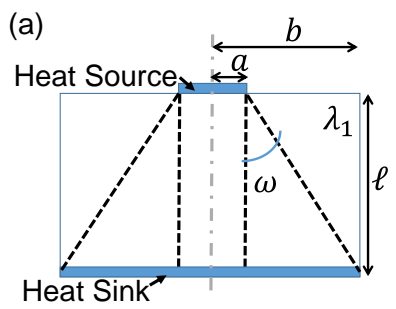

(b)

(c)

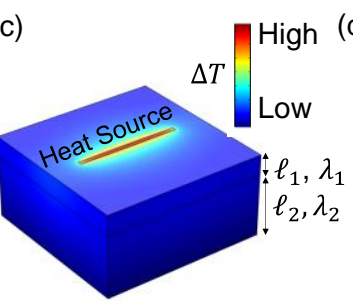

(d)

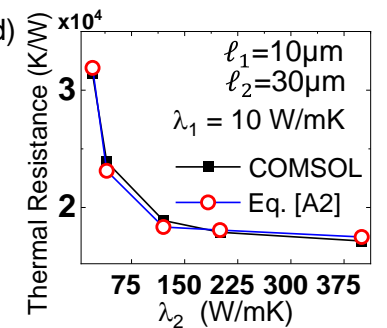

Fig. A1. (a) Schematic of a bulk substrate, with a spreading angle, $\omega$ and thermal conductivity, $\lambda$. (b) Schematic of a bilayer substrate, with a spreading angle, $\omega_{1}$ and $\omega_{2}$, thermal conductivity, $\lambda_{1}$ and $\lambda_{2}$, and widths $\ell_{1}$ and $\ell_{2}$, for the top and bottom layers, respectively. (c) COMSOL simulation to obtain $\theta_{t h}$, of a bilayer structure. Here, Fourier Equation, $q=-\kappa \nabla T$, has been solved. Power of $1 \mathrm{~W}$ has been applied of the structure and $\theta_{t h}=\Delta T / P(\mathrm{~d})$ Comparison of Eq. (A2), with 3D COMSOL simulation.

$$
\theta_{\text {th, bilayer }}=\frac{1}{\lambda_{1} \pi a} \frac{\ell_{1}+\left(\ell_{2} \times \lambda_{1} / \lambda_{2}\right)}{\left(a+\left(\ell_{1}+\ell_{2}\right) \tan \omega\right)}
$$

Eq.(A2) provides a reasonable approximation of $\theta_{\text {th }}$ as confirmed by $3 D$ COMSOL simulations, shown in Fig Al(d). Even though the range of $\lambda_{2}$ is much larger than $\lambda_{1}, \theta_{t h}$ is accurately reproduced using Eq. (A2). It is to be noted that Eq. (A2) should only be used for back-of-the-hand approximations of $\theta_{t h}$ and not as a replacement of bilayer $\theta_{t h}$ determining closed-form formulas, numerical expressions, or TCAD.

\section{B. 3D Thermal Resistance Model}

A more sophisticated analytical model, originally developed by Darwish et al. [61], considers devices with much wider gate width compared to gate length $\left(W_{g} \gg L_{g}\right)$. There are three components of the thermal resistance (labeled in subscripts $I$, $I I$, and $I I I$, with $\left.\theta_{t h, t o t}=\theta_{t h, I}+\theta_{t h, I I}+\theta_{t h, I I I}\right)$, for the active layer, and upper/lower part of the substrate. Due to the prolonged gate width (hence the heat source), instead of a spherical heat spreading, a spheroid spreading is expected. With reasonable accuracy, we simplify the original thermal resistance model in [61] as follows:

$$
\begin{gathered}
\theta_{t h, I}=\frac{1}{\pi W_{g} \lambda_{c h}} \ln \left(\frac{4 D_{c h}}{\pi L_{g}}\right) . \\
\theta_{t h, I I}=\frac{1}{\pi W_{g} \lambda_{s u b}} \ln \left(\frac{r_{A}^{I I}-1}{r_{B}^{I I}-1}\right) . \\
\theta_{t h, I I I}=\frac{1}{2 \pi s \lambda_{s u b}} \ln \left(\frac{r_{B}^{I I I}+1}{r_{B}^{I I I}-1}\right) .
\end{gathered}
$$

where $r_{A}^{I I}=\frac{\pi^{2} W_{g}}{8 D_{c h}} \frac{\lambda_{s u b}}{\lambda_{c h}}, r_{B}^{I I}=\sqrt{\left(\frac{W_{g}}{2}\right)^{2}-\left(\frac{4 \lambda_{c h} D_{c h}}{\pi^{2} \lambda_{s u b}}\right)+\left(\frac{S}{\sqrt{2}}\right)^{2}} /$ $\frac{s}{\sqrt{2}}$, and $r_{B}^{I I I}=\sqrt{\left(\frac{W_{g}}{2}\right)^{2}+\left(\frac{\pi D_{s u b}}{2}\right)^{2}} / \frac{\pi D_{\text {sub }}}{2}$ and $s$ is the length for adiabatic boundary conditions.

\section{Transient Thermal Response of a Packaged chip}

It is extremely important to calculate the transient thermal response of the chip accurately to determine the $i$-SOA. The junction temperature can be approximated by the superposition of the heating and cooling responses of the chip. For a train of repetitive pulses, the power pulses can be averaged, and the temperature calculated at the end of $n$th or $(n+1)$ th pulse after the average power pulse. The expression is given by [62]:

$$
Z_{1}=D+(1-D) \mathrm{Z}_{0}(D T)
$$

where $D$ is the duty cycle, $Z_{0}$ is the transient thermal impedance measured experimentally. For a single pulse, $\mathrm{Z}_{0}\left(t_{o n}\right)=1-\exp \left(t_{o n} / \tau_{t h}\right)$, with $\tau_{t h}\left(\equiv \theta_{t h} C_{t h}\right)$ and $T$ is the period. Another approach is to calculate the temperature at the end of the average power pulse (before steady-state conditions), where the average power is modified by a transient thermal resistance factor, given as [62]: 


$$
Z_{2}=D+(1-D) Z_{0}(D+1) T+Z_{0}(D T)-Z_{0}(T)
$$

While both $\mathrm{A} 6$ and $\mathrm{A} 7$ provide a reasonable estimation of transient thermal response, they were derived using the superposition principle, since it defies a solution otherwise. Here, we will derive a closed-form analytical relation to obtain the transient thermal response of a packaged chip, and compare it with those obtained using the superposition principle. We know from [17] that, $T_{j, \max }^{p k}-T_{a m b}=\Delta T_{L}^{p k}$ and $P^{p k}=P_{\max }$. Also,

$$
\Delta T_{L}^{p k}=P_{\text {avg }} \theta_{\text {th }}+H\left(P^{p k}-P_{\text {avg }}\right)=P^{p k} R \theta_{t h}\left[D+(1-D) H_{0}\right] .
$$

Therefore, we can write:

$$
Z=\frac{\Delta T_{L}^{p k}}{\theta_{t h} P_{\max }}=\frac{\Delta T_{L}^{p k}}{\theta_{t h^{P^{p k}}}}=D+(1-D) H_{0} .
$$

where $H_{0}=\left(1-e^{\left(-\frac{D \zeta}{\tau_{t h}}\right)}\right)\left(1-e^{\left(-\frac{(1-D) \zeta}{\tau_{t h}}\right)}\right) /\left(1-e^{\left(-\frac{\zeta}{\tau_{t h}}\right)}\right)=C Z_{0}$. For a single-time-constant system, $H_{0}$ in [52] is exact, therefore missing the infinite series contribution, i.e,

$$
C=H_{0} / Z_{0}(t)=1-e^{\left(-\frac{(1-D) \zeta}{\tau_{t h}}\right)} / 1-e^{\left(-\frac{\zeta}{\tau_{t h}}\right)} .
$$

From [18], for a packaged device, if $\tau_{t h, 1} \ll \tau_{t h, 2} \ll$ $\ldots \tau_{t h, n}, \Delta T$ of successive stages can be added, so that $\Delta T(t)=$ $\sum_{i} \Delta T_{i}\left(1-\exp \left(-\frac{t}{\tau_{t h, i}}\right)\right)$. Now dividing both sides with the dissipated power, $P_{D}$, we get:

$$
\frac{\Delta T(t)}{P_{D}}=\sum_{i} \frac{\Delta T_{i}}{P_{D}} Z_{0, i}(t) \Rightarrow Z_{0}(t)=\sum_{i} \frac{\theta_{t h, i}}{\theta_{t h, t o t}} Z_{0, i}(t) .
$$

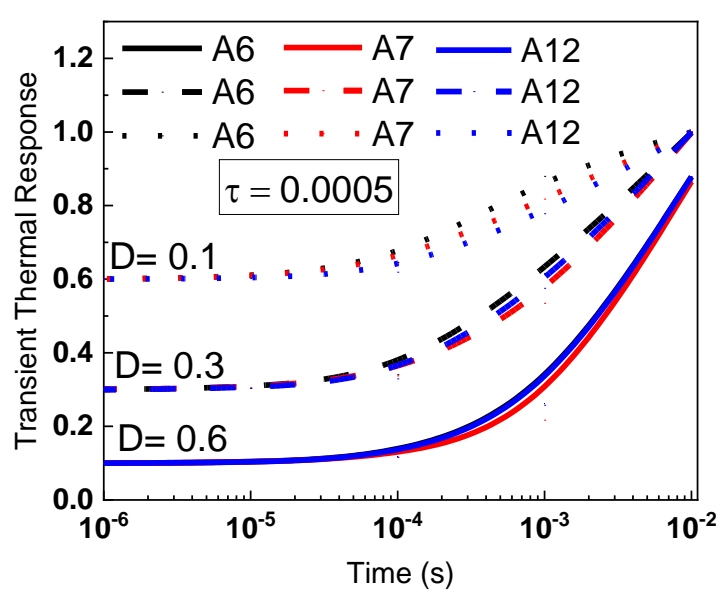

Fig. A2. Comparison of expressions for transient thermal responses of a packaged chip derived using superposition principle $\left(Z_{1}\right.$ (Eq. (A6)) and $Z_{2}$ (Eq. (A7))) and the closed form analytical solution derived in this paper $\left(Z_{m}\right.$ (Eq. (A12)). For the comparison, the thermal time constant, $\tau$ was taken as 0.0005 , which is a typical value for microchips. Although the curves are obtained for three values of duty cycles $(D=0.1,0.3$ and 0.6$)$, matching of $Z_{3}$ with $Z_{1}$ and $Z_{2}$ has been confirmed at other values of duty cycles and thermal time constants as well.

where $Z_{0}(t)$ is the average of all individual stage $Z_{0, i}(t)$, and $\theta_{t h, t o t}$ is the summation of all $\theta_{t h}$ of the packaged chip.
Therefore, a multi-stage transient thermal resistance (Eq. (A11)) including the correction factor $C(D, \zeta)$ (Eq. (A10)) and duty cycle $D$ (Eq. (A9)) can be expressed as:

$$
Z_{m}(t)=D+(1-D) \sum_{i} \frac{\theta_{t h, i}}{\theta_{t h, t o t}} C_{i}(D, \zeta) Z_{0, i}(t)
$$

Fig. A2 shows the derived analytical closed-form solution of transient thermal response, $Z_{m}$ (Eq. (A12)) matches the responses predicted by $Z_{1}$ (Eq. $\left.\mathrm{A}(6)\right)$ and $Z_{2}$ (Eq. $\left.\mathrm{A}(7)\right)$ very well.

\section{Device Geometry and Parameters Used for Calculations.}

Table T1: Device Dimensions used in calculations.

\begin{tabular}{|c|c|c|c|c|c|}
\hline Material & $\mathrm{Si}$ & $\mathrm{SiC}$ & $\mathrm{GaN}$ & $\beta-\mathrm{Ga}_{2} \mathrm{O}_{3}$ & Diamond \\
\hline Bandgap (eV) & 1.1 & 3.25 & 3.4 & 4.85 & 5.47 \\
\hline Dielectric Const. & 11.8 & 9.7 & 9 & 10 & 5.5 \\
\hline $\begin{array}{c}\text { Thermal Cond. } \\
(\mathrm{W} / \mathrm{cmK})\end{array}$ & 1.5 & 4.9 & 2.3 & 0.2 & 20 \\
\hline $\begin{array}{c}\text { Electron Mobility } \\
\left(\mathrm{cm}^{2} / \mathrm{Vs}\right)\end{array}$ & 1480 & 1000 & 1250 & 300 & 2000 \\
\hline $\begin{array}{c}\text { Breakdown } \\
\text { Field (MV/cm) }\end{array}$ & 0.3 & 2.5 & 3.3 & 8 & 10 \\
\hline Sp. Heat (J/K-g) & 0.7 & 0.69 & 0.49 & 0.49 & 0.52 \\
\hline Density (g/cm $\left.{ }^{3}\right)$ & 2.33 & 3.1 & 6.15 & 5.95 & 3.52 \\
\hline $\begin{array}{c}C_{t h} / W(\mathrm{~J} / \mathrm{K}-\mathrm{cm}) \\
\left(\times 10^{-5}\right)\end{array}$ & 4.89 & 642 & 904 & 875 & 549 \\
\hline
\end{tabular}

Table T2: Device Parameters used in calculations.

\begin{tabular}{|c|c|c|c|c|}
\hline & Layers & Length & Width & Thickness \\
\hline Bulk Substrate & Bulk & $4 \mu \mathrm{m}$ & $50 \mu \mathrm{m}$ & $300 \mu \mathrm{m}$ \\
\hline Bilayer & Layer 1 & $4 \mu \mathrm{m}$ & $50 \mu \mathrm{m}$ & $5 \mu \mathrm{m}$ \\
\cline { 2 - 5 } Structure & Layer 2 & $4 \mu \mathrm{m}$ & $50 \mu \mathrm{m}$ & $300 \mu \mathrm{m}$ \\
\hline
\end{tabular}

\section{REFERENCES}

[1] P. Wilson, The Circuit Designer's Companion. Newnes, 2017.

[2] B. J. Baliga, "Semiconductors for high-voltage, vertical channel field-effect transistors," J. Appl. Phys., vol. 53, no. 3, pp. 1759-1764, 1982, doi: $10.1063 / 1.331646$.

[3] R. W. Keyes, "Figure of merit for semiconductors for high-speed switches," Proc. IEEE, vol. 60, no. 2, p. 225, 1972, doi: 10.1109/PROC.1972.8593.

[4] E. Johnson, "Physical limitations on frequency and power parameters of transistors," in 1958 IRE International Convention Record, Mar. 1965, vol. 13, pp. 27-34, doi: 10.1109/IRECON.1965.1147520.

[5] A. Q. Huang, "New unipolar switching power device figures of merit," IEEE Electron Device Lett., vol. 25, no. 5, pp. 298-301, 2004, doi: 10.1109/LED.2004.826533.

[6] K. Shenai, "The Figure of Merit of a Semiconductor Power Electronics Switch," IEEE Trans. Electron Devices, vol. 65, no. 10, pp. 4216-4224, Oct. 2018, doi: 10.1109/TED.2018.2866360. K. D. Chabak et al., "Lateral \$lupbeta-Ga2O3 field effect transistors," Semicond. Sci. Technol., vol. 35, no. 1, p. 13002, Nov. 2019, doi: 10.1088/1361-6641/ab55fe.

[8] S. J. Pearton et al., "A review of $\mathrm{Ga} 2 \mathrm{O} 3$ materials, processing, and devices," Appl. Phys. Rev., vol. 5, no. 1, p. 11301, Jan. 2018, doi: 10.1063/1.5006941.

[9] H. Zhou, M. Si, S. Alghamdi, G. Qiu, L. Yang, and P. D. Ye, "HighPerformance Depletion/Enhancement-ode $\$ \beta \$-\mathrm{Ga} 2 \mathrm{O} 3$ on Insulator (GOOI) Field-Effect Transistors With Record Drain Currents of 600/450 mA/mm," IEEE Electron Device Lett., vol. 38, no. 1, pp. 103-106, Jan. 2017, doi: 10.1109/LED.2016.2635579.

[10] B. K. Mahajan, Y.-P. Chen, J. Noh, P. D. Ye, and M. A. Alam, "Electrothermal performance limit of $\beta-\mathrm{Ga} 2 \mathrm{O} 3$ field-effect transistors," Appl. Phys. Lett., vol. 115, no. 17, p. 173508, Oct. 2019, 
doi: $10.1063 / 1.5116828$

[11] H. Zhou, K. Maize, J. Noh, A. Shakouri, and P. D. Ye, "Thermodynamic Studies of $\beta-G a 2 \mathrm{O} 3$ Nanomembrane Field-Effect Transistors on a Sapphire Substrate," ACS Omega, vol. 2, no. 11, pp. 7723-7729, Nov. 2017, doi: 10.1021/acsomega.7b01313.

[12] G. Meneghesso et al., "Reliability of GaN High-Electron-Mobility Transistors: State of the Art and Perspectives," IEEE Trans. Device Mater. Reliab., vol. 8, no. 2, pp. 332-343, Jun. 2008, doi: 10.1109/TDMR.2008.923743

[13] B. K. Mahajan, Y. Chen, W. Ahn, N. Zagni, and M. A. Alam, "Design and Optimization of \$lboldsymbol \{ \beta\} \$-Ga2O3 on (h-BN layered) Sapphire for High Efficiency Power Transistors: A Device-CircuitPackage Perspective," in 2018 IEEE International Electron Devices Meeting (IEDM), 2018, pp. 24.6.1-24.6.4, doi: 10.1109/IEDM.2018.8614714.

[14] U. Radhakrishna, T. Imada, T. Palacios, and D. Antoniadis, "MIT virtual source GaNFET-high voltage (MVSG-HV) model: A physics based compact model for HV-GaN HEMTs," Phys. status solidi c, vol. 11, no. 3-4, pp. 848-852, Feb. 2014, doi: 10.1002/pssc.201300392.

[15] N. Zagni et al., "'Hole Redistribution' Model Explaining the Thermally Activated RON Stress/Recovery Transients in CarbonDoped AlGaN/GaN Power MIS-HEMTs," IEEE Trans. Electron Devices, vol. 68, no. 2, pp. 697-703, Feb. 2021, doi: 10.1109/TED.2020.3045683.

[16] S. H. Shin et al., "Fundamental trade-off between short-channel control and hot carrier degradation in an extremely-thin silicon-oninsulator (ETSOI) technology," in 2015 IEEE International Electron Devices Meeting (IEDM), 2015, pp. 20.3.1-20.3.4, doi: 10.1109/IEDM.2015.7409741.

[17] H. Jiang, S. Shin, X. Liu, X. Zhang, and M. A. Alam, “The Impact of Self-Heating on HCI Reliability in High-Performance Digital Circuits," IEEE Electron Device Lett., vol. 38, no. 4, pp. 430-433, 2017, doi: 10.1109/LED.2017.2674658.

[18] Q. Shan, Q. Dai, S. Chhajed, J. Cho, and E. F. Schubert, "Analysis of thermal properties of GaInN light-emitting diodes and laser diodes," J. Appl. Phys., vol. 108, no. 8, p. 84504, Oct. 2010, doi: 10.1063/1.3493117.

[19] B. K. Mahajan, Y. Chen, D. Varghese, S. Krishnan, V. Reddy, and M. A. Alam, "Quantifying Region-Specific Hot Carrier Degradation in LDMOS Transistors Using a Novel Charge Pumping Technique," in 2021 IEEE International Reliability Physics Symposium (IRPS), 2021, pp. 1-6.

[20] S. Mahapatra and R. Saikia, "On the Universality of Hot Carrier Degradation: Multiple Probes, Various Operating Regimes, and Different MOSFET Architectures," IEEE Trans. Electron Devices, vol. 65, no. 8, pp. 3088-3094, 2018, doi: 10.1109/TED.2018.2842129.

[21] B. K. Mahajan, Chen, Y.-P. Chen, and M. A. Alam, "An Analytical Model of Hot Carrier Degradation in LDMOS Transistors: Rediscovery of Universal Scaling," IEEE Trans. Electron Devices, $68,8,2021$.

[22] Alam, Muhammad A, Mahajan, Bikram K., and Y.-P. Cheri, "Hot carrier Degradation in Classical and Emerging Logic and Power Electronic Devices: Rethinking Reliability for Next-Generation Electronics," in 5th IEEE Electron Devices Technology and Manufacturing Conference, 2021, pp. 1-3.

[23] M. S. Adler and S. R. Westbrook, "Power semiconductor switching devices-A comparison based on inductive switching," IEEE Trans. Electron Devices, vol. 29, no. 6, pp. 947-952, Jun. 1982, doi: 10.1109/T-ED.1982.20811.

[24] T. Kimoto and J. A. Cooper, Fundamentals of Silicon Carbide Technology: Growth, Characterization, Devices and Applications, vol. 9781118313.2014.

[25] N. D. Arora, J. R. Hauser, and D. J. Roulston, "Electron and hole mobilities in silicon as a function of concentration and temperature," IEEE Trans. Electron Devices, vol. 29, no. 2, pp. 292-295, Feb. 1982, doi: 10.1109/T-ED.1982.20698.

[26] H. Umezawa, M. Nagase, Y. Kato, and S. I. Shikata, "High temperature application of diamond power device," Diam. Relat. Mater., vol. 24, pp. 201-205, 2012, doi: 10.1016/j.diamond.2012.01.011. "http://www.ioffe.ru/SVA/NSM/Semicond/."Accesed: 31-Aug-2021.
Phys., vol. 109, no. 3, p. 34501, 2011, doi: 10.1063/1.3524185.

M. J. Tadjer, J. L. Lyons, N. Nepal, J. A. Freitas, A. D. Koehler, and G. M. Foster, "Theory and Characterization of Doping and Defects in \$lupbeta\$-Ga2O3," \{ECS\} J. Solid State Sci. Technol., vol. 8, no. 7, pp. Q3187--Q3194, 2019, doi: 10.1149/2.0341907jss.

[30] P. Gorai, R. W. McKinney, N. M. Haegel, A. Zakutayev, and V. Stevanovic, "A computational survey of semiconductors for power electronics," Energy Environ. Sci., vol. 12, no. 11, pp. 3338-3347, 2019, doi: 10.1039/C9EE01529A.

[31] M. Faqir, G. Verzellesi, G. Meneghesso, E. Zanoni, and F. Fantini, "Investigation of High-Electric-Field Degradation Effects in AlGaN/GaN HEMTs," IEEE Trans. Electron Devices, vol. 55, no. 7, pp. 1592-1602, Jul. 2008, doi: 10.1109/TED.2008.924437.

[32] N. Donato, N. Rouger, J. Pernot, G. Longobardi, and F. Udrea, "Diamond power devices: state of the art, modelling, figures of merit and future perspective," J. Phys. D. Appl. Phys., vol. 53, no. 9, p. 93001, Dec. 2019, doi: 10.1088/1361-6463/ab4eab.

[33] J. Oh, J. Ma, and G. Yoo, "Simulation study of reduced self-heating in $\beta-G a 2 O 3$ MOSFET on a nano-crystalline diamond substrate," Results Phys., vol. 13, p. 102151, 2019, doi: https://doi.org/10.1016/j.rinp.2019.02.087.

[34] S. Strauss, A. Erlebach, T. Cilento, D. Marcon, S. Stoffels, and B. Bakeroot, "TCAD methodology for simulation of GaN-HEMT power devices," in 2014 IEEE 26th International Symposium on Power Semiconductor Devices IC's (ISPSD), Jun. 2014, pp. 257-260, doi: 10.1109/ISPSD.2014.6856025.

[35] H. Ding, K. Isoird, H. Schneider, S. Kone, and G. Civrac, "Basic parameters and models in simulation of CVD diamond devices," Diam. Relat. Mater., vol. 19, no. 5, pp. 500-502, 2010, doi: https://doi.org/10.1016/j.diamond.2009.12.014.

[36] C. J. H. Wort and R. S. Balmer, "Diamond as an electronic material," Mater. Today, vol. 11, no. 1, pp. 22-28, 2008, doi: https://doi.org/10.1016/S1369-7021(07)70349-8.

[37] J. Noh et al., "High Performance $\$ \beta \$-G a 2 O 3$ Nano-Membrane Field Effect Transistors on a High Thermal Conductivity Diamond Substrate," IEEE J. Electron Devices Soc., vol. 7, pp. 914-918, 2019, doi: 10.1109/JEDS.2019.2933369.

[38] D. Lei, K. Han, Y. Wu, Z. Liu, and X. Gong, "High Performance Ga2O3 Metal-Oxide-Semiconductor Field-Effect Transistors on an AlN/Si Substrate," IEEE J. Electron Devices Soc., vol. 7, pp. 596600, 2019, doi: 10.1109/JEDS.2019.2915341.

[39] N. Ikeda et al., "GaN Power Transistors on Si Substrates for Switching Applications," Proc. IEEE, vol. 98, no. 7, pp. 1151-1161, Jul. 2010, doi: 10.1109/JPROC.2009.2034397.

[40] K. D. Chabak et al., "Recessed-Gate Enhancement-Mode $\$ \beta \$$ Ga2O3 MOSFETs," IEEE Electron Device Lett., vol. 39, no. 1, pp. 67-70, Jan. 2018, doi: 10.1109/LED.2017.2779867.

[41] E. Pop, "Energy dissipation and transport in nanoscale devices," Nano Res., vol. 3, no. 3, pp. 147-169, 2010, doi: 10.1007/s12274010-1019-z.

[42] M. H. Wong, Y. Morikawa, K. Sasaki, A. Kuramata, S. Yamakoshi, and M. Higashiwaki, "Characterization of channel temperature in $\mathrm{Ga} 2 \mathrm{O} 3$ metal-oxide-semiconductor field-effect transistors by electrical measurements and thermal modeling," Appl. Phys. Lett., vol. 109 , no. 19 , p. 193503, Nov. 2016, doi: 10.1063/1.4966999.

[43] M. Singh et al., "Pulsed Large Signal RF Performance of Field-Plated Ga2O3 MOSFETs," IEEE Electron Device Lett., vol. 39, no. 10, pp. 1572-1575, 2018, doi: 10.1109/LED.2018.2865832.

[44] J. W. Pomeroy et al., "Raman Thermography of Peak Channel Temperature in \$\$ $\$$-Ga2O3 MOSFETs," IEEE Electron Device Lett., vol. 40, no. 2, pp. 189-192, 2019, doi: 10.1109/LED.2018.2887278.

[45] S. Sandell, E. Chávez-Ángel, A. El Sachat, J. He, C. M. Sotomayor Torres, and J. Maire, "Thermoreflectance techniques and Raman thermometry for thermal property characterization of nanostructures," J. Appl. Phys., vol. 128, no. 13, p. 131101, Oct. 2020, doi: 10.1063/5.0020239.

[46] K. Shenai, "High-Density Power Conversion and Wide-Bandgap Semiconductor Power Electronics Switching Devices," Proc. IEEE, vol. 107, no. 12, pp. 2308-2326, 2019, doi: 10.1109/JPROC.2019.2948554.

[47] K. Shenai, "(Invited) Thermal Limitations in Wide Bandgap ( $\{\mathrm{WBG}\})$ Semiconductor Power Switching Devices," $\{$ ECS $\}$ Trans., vol. 66, no. 1, pp. 53-65, May 2015, doi: 10.1149/06601.0053ecst.

[48] P. Moens and G. Van Den Bosch, "Characterization of Total Safe Operating Area of Lateral DMOS Transistors," IEEE Trans. Device degradation in GaN high-electron-mobility transistors," J. Appl. 
Mater. Reliab., vol. 6, no. 3, pp. 349-357, 2006, doi: 10.1109/TDMR.2006.882212.

[49] P. L. Hower and S. Pendharkar, "Short and long-term safe operating area considerations in LDMOS transistors," in 2005 IEEE International Reliability Physics Symposium, 2005. Proceedings. 43rd Annual., 2005, pp. 545-550, doi: 10.1109/RELPHY.2005.1493145.

[50] D. Brisbin, P. Lindorfer, and P. Chaparala, "Anomalous Safe Operating Area and Hot Carrier Degradation of NLDMOS Devices," IEEE Trans. Device Mater. Reliab., vol. 6, no. 3, pp. 364-370, 2006, doi: 10.1109/TDMR.2006.883558.

[51] S. C. Sun and J. D. Plummer, "Electron mobility in inversion and accumulation layers on thermally oxidized silicon surfaces," IEEE Trans. Electron Devices, vol. 27, no. 8, pp. 1497-1508, Aug. 1980, doi: 10.1109/T-ED.1980.20063.

[52] K. Hess et al., "Theory of channel hot-carrier degradation in MOSFETs," Phys. B Condens. Matter, vol. 272, no. 1, pp. 527-531, 1999, doi: https://doi.org/10.1016/S0921-4526(99)00363-4.

[53] M. A. Alam, B. K. Mahajan, Y. Chen, W. Ahn, H. Jiang, and S. H. Shin, "A Device-to-System Perspective Regarding Self-Heating Enhanced Hot Carrier Degradation in Modern Field-Effect Transistors: A Topical Review," IEEE Trans. Electron Devices, vol. 66, no. 11, pp. 4556-4565, 2019, doi: 10.1109/TED.2019.2941445.

[54] S. Mahapatra and U. Sharma, "A Review of Hot Carrier Degradation in n-Channel MOSFETs - Part I: Physical Mechanism," IEEE Trans. Electron Devices, vol. 67, no. 7, pp. 2660-2671, Jul. 2020, doi: 10.1109/TED.2020.2994302.

[55] G. V Groeseneken, "Hot carrier degradation and ESD in submicrometer CMOS technologies: how do they interact?," IEEE Trans. Device Mater. Reliab., vol. 1, no. 1, pp. 23-32, Mar. 2001, doi: 10.1109/7298.946457.

[56] P. Heremans, G. Van den Bosch, R. Bellens, G. Groeseneken, and H. E. Maes, "Temperature dependence of the channel hot-carrier degradation of n-channel MOSFET's," IEEE Trans. Electron Devices, vol. 37, no. 4, pp. 980-993, Apr. 1990, doi: $10.1109 / 16.52433$.

[57] B. Kaczer et al., "Ubiquitous relaxation in BTI stressing-New evaluation and insights," in 2008 IEEE International Reliability Physics Symposium, Apr. 2008, pp. 20-27, doi: 10.1109/RELPHY.2008.4558858.

[58] J. Joh, F. Gao, T. Palacios, and J. A. del Alamo, "A model for the critical voltage for electrical degradation of GaN high electron mobility transistors," Microelectron. Reliab., vol. 50, no. 6, pp. 767773, 2010, doi: https://doi.org/10.1016/j.microrel.2010.02.015.

[59] Y. Chen, B. K. Mahajan, D. Varghese, S. Krishnan, V. Reddy, and M. A. Alam, "A Novel 'I-V Spectroscopy' Technique to Deconvolve Threshold Voltage and Mobility Degradation in LDMOS Transistors," in 2020 IEEE International Reliability Physics Symposium (IRPS), Apr. 2020, pp. 1-6, doi: 10.1109/IRPS45951.2020.9128965.

[60] F. N. Masana, "A closed form solution of junction to substrate thermal resistance in semiconductor chips," IEEE Trans. Components, Packag. Manuf. Technol. Part A, vol. 19, no. 4, pp. 539-545, Dec. 1996, doi: 10.1109/95.554935.

[61] A. M. Darwish, A. J. Bayba, and H. A. Hung, "Thermal resistance calculation of AlGaN-GaN devices," IEEE Trans. Microw. Theory Tech., vol. 52, no. 11, pp. 2611-2620, Nov. 2004, doi: 10.1109/TMTT.2004.837200.

[62] B. Roehr and B. Shiner, "Transient Thermal Resistance - General Data and Its Use, On Semiconductor, AN569/D," 2001.

[63] N. Oi et al., "Vertical-type two-dimensional hole gas diamond metal oxide semiconductor field-effect transistors," Sci. Rep., vol. 8, no. 1, p. 10660 , Dec. 2018, doi: 10.1038/s41598-018-28837-5.

[64] E. Gheeraert, "Fundamental material's nature of diamond," in Power Electronics Device Applications of Diamond Semiconductors, Elsevier, 2018, pp. 191-217. 